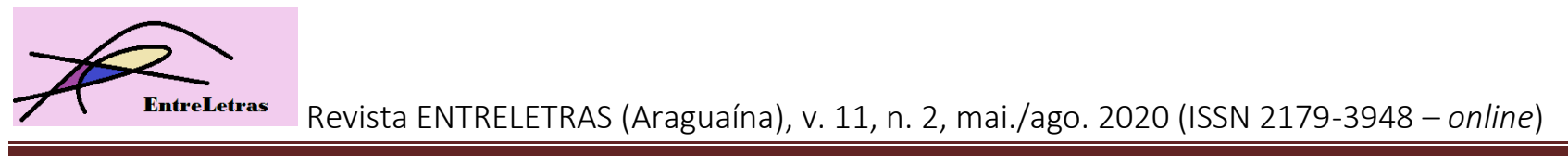

\title{
FANON Y LITERATURA: \\ L'OEIL SE NOIE UN PRÓLOGO A PEAU NOIRE, MASQUES BLANCS
}

\author{
FANON AND LITERATURE: \\ L'OEIL SE NOIE AS A PROLOGUE TO PEAU NOIRE, MASQUES BLANCS
}

Vialcary Crisóstomo Tejada ${ }^{1}$

\begin{abstract}
Resumen: Frantz Fanon es uno de los más importantes filósofos y psiquiatras del siglo XX. Aunque los textos políticos y psiquiátricos de Fanon se encuentran entre los más estudiados, sus obras literarias hasta recientemente no habían sido publicadas. En el volumen Alienation and Freedom (2018), los editores Jean Khalfa y Robert J.C. Young publican por primera vez dos obras de teatro fanonianas: L'Oeil se noie y Les Mains parallèles. En este ensayo analizo L'Oeil se noie en relación a las teorías de colonialismo y raza que Fanon desarrolla a lo largo de sus textos y sugiero que esta obra literaria indaga en los efectos psicológicos del colonialismo y la racialización. Propongo que L'Oeil se noie es un análisis existencial en el cual el autor articula la alienación del sujeto colonial/racializado. Partiendo desde un análisis de las teorías de neurosis de abandono y sociogenia discutidas en Peau noire, masques blancs (1952), argumento que en esta obra de teatro Fanon analiza el colonialismo como causante de una psicosis identitaria que enajena al sujeto racializado de la realidad en un mundo antinegro.
\end{abstract}

Palabras claves: Colonialismo; racialización; neurosis de abandono; sociogenia; mundo antinegro

\begin{abstract}
Frantz Fanon is one of the most important philosophers and psychiatrists of the $20^{\text {th }}$ century. Although Fanon's political and psychiatric work are among the most studied, his literary texts had not been published until recently. In the volume Alienation and Freedom (2018), editors Jean Khalfa and Robert J.C. Young published for the first time two Fanonian plays: L'Oeil se noie and Les Mains parallèle. In this essay, I analyze L'Oeil se noie in relation to the theories of colonialism and race discussed throughout Fanon's texts, and argue that this literary text explores the psychological effects of colonialism and racialization. I propose that L'Oeil se noie is an existential analysis, in which the author articulates the alienation of the colonial/racialized subject. Borrowing from the theories of abandonment neurosis and sociogeny discussed in Peau noire, masques blancs (1952), I argue that in this play Fanon analyzes colonialism as the cause of an identity psychosis that alienates black subjects from the reality of an anti-black world.
\end{abstract}

Keywords: Colonialism: racialization; abandonment neurosis; sociogeny; anti-black world

\footnotetext{
${ }^{1}$ Vialcary Crisóstomo Tejada, Ph.D is an Assistant Professor of Spanish and Hispanic Studies at Earlham College. Her research focuses on Caribbean literature, specifically on racial and gender policies on the Dominican Republic. Her research interests are on race and gender discourses, and migration policies in the Spanish Caribbean. Email: crisovi@earlham.edu
} 


\section{Introducción}

Desde la publicación de Peau noire, masques blancs (Black Skin, White Masks²) en 1952, las obras políticas y filosóficas de Frantz Fanon se encuentran entre las más influyentes de nuestros tiempos. A pesar de su popularidad como revolucionario y filósofo, muchos de los escritos de Fanon hasta recientemente no habían sido publicados o traducidos. En el volumen Alienation and Freedom (2018), Jean Khalfa y Robert J.C. Young reúnen textos psiquiátricos, políticos y literarios inéditos de Fanon. En esta colección Khalfa y Young presentan nuevas facetas de Fanon como psiquiatra y escritor, y su compromiso político ante un mundo antinegro. El volumen inicia con dos obras de teatro, L'Oeil se noie (The Drowning Eye) y Les Mains parallèles (Parallel Hands), los primeros textos que escribe Fanon. Dichas obras aún no han sido analizadas extensivamente por la crítica literaria fanoniana. En este ensayo analizo la primera obra de la colección, L'Oeil se noie (1949) y argumento que en ella Fanon discute dos de los principales conceptos que tres años más tarde desarrolla en su canónico texto Peau noire: neurosis de abandono y sociogenia.

\section{Peau noire, masques blancs: Entre sociogenia y neurosis de abandono}

En 1952, Frantz Fanon publica su primer texto Peau noire, masques blancs. En esta canónica obra Fanon argumenta que el racismo antinegro que existe en Francia, y en otras naciones coloniales, es un estado psicológico que se produce como resultado de una realidad alienante que normaliza la identidad blanca y deshumaniza la identidad negra. Mediante un texto creolizado $^{3}$, que reúnen experiencias personales, análisis literario, estudios psiquiátricos y teorías filosóficas, Fanon analiza la experiencia negra en una sociedad racista. Peau noire explora la relación que existe entre nociones de raza, construcciones sociales y problemas psicológicos. Según Fanon, la construcción colonial de un mundo antinegro demanda que los sujetos negros sean blancos para poder ser concebidos como humanos. La imposibilidad del sujeto negro de convertirse en blanco produce un estado psicológico de alienación. En este texto, Fanon presenta un diagnóstico psicológico al complejo de inferioridad que la retórica antinegra produce en el sujeto negro colonial. Al no ser reconocido como humano en un mundo antinegro, el sujeto racializado pierde

\footnotetext{
${ }^{2}$ En este ensayo estaré usando traducciones al inglés de los textos de Fanon.

3 Para conocer más de la teoría de criollización véase Creolizing Theory de Jane A. Gordon
} 
su agencia, lo cual conduce a la enajenación del sujeto negro. Peau noire es principalmente un estudio psicológico que analiza la salud mental del sujeto negro y propone un diagnóstico social para la psicosis que produce el racismo.

Fanon introduce dos importantes tesis para entender el estado psicológico que el racismo produce en el sujeto negro; neurosis de abandono y sociogenia. El autor discute el concepto de neurosis de abandono que inicialmente desarrolla Germaine Guex. Según Guex, "Affective selfrejection invariably brings the abandonment-neurotic to an extremely painful and obsessive feeling of exclusion, of having no place anywhere, of being superfluous everywhere in an affective sense" (Fanon, 2008, p.55). Partiendo desde el concepto de Guex, Fanon argumenta que el sujeto negro sufre de neurosis de abandono ya que socialmente ha sido abandonado por una sociedad antinegra que lo deshumaniza. En un mundo antinegro la negritud es ininteligible y, por lo tanto, el negro no es reconocido como humano. Fanon afirma que el mundo antinegro sitúa al sujeto negro en la zona del no-ser, dicho posicionamiento significa que "the black is not a man" (Fanon, 2008, p.1). Al no ser reconocido como humano, el sujeto negro sufre de un complejo de inferioridad que se desarrolla en una neurosis de abandono. De acuerdo a Guex:

This lack of esteem of self as an object worthy of love has grave consequences. For one thing, it keeps the individual in a state of profound inner insecurity, as a result of which it inhibits or falsifies every relation with others. It is as something that has the right to arouse sympathy or love that the individual is uncertain of himself. The lack of affective selfvaluation is to be found only in persons who in their early childhood suffered from a lack of love and understanding (FANON, 2008, p.55).

Fanon argumenta que la ausencia de autoestima afecta particularmente al sujeto negro que es articulado como no-humano por el discurso antinegro. Esta ausencia de autoestima y el desarrollo de inseguridades, conducen al sujeto negro a una neurosis de abandono que sirve como mecanismo de defensa en un mundo que rechaza su humanidad.

A pesar de la neurosis que el mundo antinegro produce en el sujeto racializado, Fanon argumenta que la solución no es la terapia individual ya que la neurosis del sujeto negro no es producto de la psicología personal sino de la jerarquización de humanidades que produce el sistema colonial moderno. Fanon propone la noción de sociogenia, la cual argumenta que la formación del sujeto no se produce solo en el ámbito biológico sino también en el social. El teórico explica: "It will be seen that the black man's alienation is not an individual question. Beside phylogeny and 
ontogeny stands sociogeny. In one sense, conforming to the view of Leconte and Damey, let us say that this is a question of a sociodiagnostic" (FANON, 2008, p.4). Al contrario de otros psiquiatras, entre ellos Freud, quienes argumentan que procesos biológicos como la filogenia y la ontogenia produce al sujeto, Fanon argumenta a favor de la sociogenia y de los factores sociales que contribuyen a la construcción del sujeto. Implementado la noción de sociogenia, Fanon asegura que lo racial también se forja con base en dinámicas sociales que humanizan a sujetos blancos y deshumanizan a sujetos negros. El filósofo explica: "But society, unlike biochemical processes, cannot escape human influences. Man is what brings society into being. The prognosis is in the hands of those who are willing to get rid of the worm- eaten roots of the structure" (Fanon, 2008, p.4). Según Fanon, al igual que otras estructuras sociales las jerarquías raciales son producidas por personas y, por ende, pueden cambiar. La sociogenia propone un nuevo tipo de terapia que se enfoca no en cambios individuales sino en cambios sociales. La neurosis que produce el mundo antinegro, tanto en sujetos negros como blancos, demuestra no una relación disfuncional con la realidad sino más bien una realidad disfuncional en sí misma. Fanon prescribe la acción política como método de contestar el socio-diagnóstico de alienación que produce el mundo antinegro. Peau noire es un texto antirracista que indaga en los efectos psicológicos y sociales que el discurso racial/colonial ha tenido, y continúa teniendo, en el desarrollo de la psíquica negra.

\section{L'Oeil se noie: Un prólogo a Peau noire, masques blancs}

Fanon escribe L'Oeil se noie en 1949 cuando es un estudiante de medicina en Lyon. La obra tenía cinco escenas, pero solo cuatro de ellas han sido recuperadas. Esta obra narra el triángulo amoroso entre los hermanos François y Lucien, y la joven Ginette. Las escenas se desarrollan en forma de diálogos entre los personajes. Al contrario de los textos psicológicos y políticos de Fanon, esta obra de teatro no discute abiertamente el colonialismo y sus repercusiones psicológicas, politicas y raciales. A pesar de que la obra no se auto declara como un texto que investiga los efectos psicológicos del colonialismo y la racialización, mediante un análisis existencial identitario Fanon logra representar la alienación que sufre el sujeto negro colonial. En L'Oeil se noie, al igual que en Peau noire, masques blancs, Fanon analiza el colonialismo como causante de una psicosis identitaria que enajena al sujeto negro de la realidad dentro de una sociedad blanca. Argumento que en esta obra Fanon empieza a desarrollar dos de los principales conceptos presentes en Peau noire; la neurosis de abandono y la sociogenia. 
En Alienation and Freedom, Young explica que "The Drowning Eye explores psychological effect of racialization without foregrounding the social milieu that has produced them, or indeed overtly discussing the questions of race as such" (YOUNG, 2018, p.33). Concuerdo con Young, L'Oeil se noie discute las mismas nociones raciales que Fanon desarrolla a lo largo de todos sus textos. En esta obra de teatro, el escritor analiza lo racial de manera más literaria y mediante simbolismo, metáforas y discursos existenciales retrata la realidad de un mundo antinegro y las consecuencias psicológicas de la alienación racial. A pesar de su perspicaz lectura racial de L'Oeil se noie, Young argumenta que al no identificar explícitamente la raza de los personajes "Fanon puts everyone in the position of the inbetween, of being neither and both at once" (YOUNG, 2018, p.33). Aunque bien es cierto que la obra no identifica explícitamente la raza de los personajes, al contrario del argumento de Young, considero que Fanon no narra un inbetweeness racial. Sugiero que la obra representa una clara visión racial que expone las diferentes y opuestas realidades en la que vive el sujeto blanco y el sujeto negro. Mediante los personajes de François y Lucien, el autor narra los dos tipos de relaciones que se pueden establecer en un mundo colonial antinegro. Sugiero leer L'Oeil se noie como prólogo a las teorías raciales y psicológicas que Fanon más tarde desarrolla en Peau noire, particularmente en cuanto a las nociones de neurosis de abandono y sociogenia se refiere.

La obra empieza con un diálogo entre François y su amada Ginette. A lo largo de esta escena François interroga a Ginette y le pide que le diga si lo ama y que explique el por qué. François rompe el silencio de la primera escena con "Do you love me?” (FANON, 2018, p.82). A pesar que Ginette contesta "I love you" y lo repite tras cada interrogante, François no está convencido del amor de Ginette por él (FANON, 2018, p.83). El constante cuestionamiento del amor de Ginette por parte de François puede ser interpretado por muchos como una cursi escena de amor entre una pareja disfuncional. Aunque este análisis no carece de evidencias, sugiero que en esta escena Fanon retrata a un personaje que sufre de neurosis de abandono. En Peau noire Fanon explica:

The abandonment-neurotic demands proofs. He is not satisfied with isolated statements. He has no confidence. Before he forms an objective relation, he exacts repeated proofs from his partner. The essence of his attitude is "not to love in order to avoid being abandoned." The abandonment-neurotic is insatiable. That is because he claims the right to constant amends. He wants to be loved completely, absolutely and forever (FANON, 2008, p.5556). 
Al igual que el sujeto que sufre de neurosis de abandono, François necesita pruebas del amor de Ginette. François dice: "I would like you to explain to me how, why and at what moment you started to love me" (FANON, 2018, p.83). Este pedido muestra que François no tiene confianza en sí mismo, dicha ausencia de autoestima le impide creer que Ginette en verdad pueda amarlo. Guex afirma que "The abandonment-neurotic doubts whether he can be loved as he is, for he has had the cruel experience of being abandoned when he offered himself to the tenderness of others as a little child and hence without artifice" (FANON, 2008, p.56). La neurosis de abandono de François le impide al personaje aceptar el amor de Ginette sin antes cuestionarlo. Fanon sugiere que la neurosis de abandono deja al sujeto en estado de "frightfully full of doubt", estado en el cual se encuentra François (FANON, 2008, p.56).

El diálogo de esta primera escena más allá de demostrar la conflictiva relación entre François y Ginette, señala la condición psicológica de la que padece François. El personaje explica que desde su niñez se ha sentido alienado de "they"-ellos-, ya que al contrario que ellos él no entiende su lugar en el mundo (FANON, 2018, p.83). La neurosis de abandono que sufre François se asemeja a la neurosis de abandono que conforme a Fanon aflige al sujeto negro colonial. Al igual que el sujeto negro quien "internaliz[ed] -or, better, epidernaliz[ed] [his] inferiority”, François ha internalizado su inferioridad (FANON, 2008, p.4). El personaje explica que de niño quería formar parte del mundo de ellos, pero nunca logró convertirse en el hombre que ellos querían. Similar al negro que desea ser blanco, François "told himself that there was perhaps a word to be known. A word of the sort that opens doors and forces the smiles of mean men" (Fanon, 2018, p.84). Mas dicha palabra nunca se materializó y François creció en un mundo que lo rechaza y el cual no entiende. François le reclama a Ginette, "You, you really do not know what it's like not to understand. Not to understand what's happening to you! (FANON, 2018, p.84). Es dicho rechazo y la imposibilidad de entender cómo formar parte del mundo, lo que genera una neurosis de abandono en François.

Al demandar una explicación del por qué lo ama, François demuestra su inseguridad a la vez que exige entender si Ginette lo humaniza a él o a una versión de él que corresponde al discurso de ellos. La neurosis de abandono de François inicialmente lo conlleva a desvalorizarse a sí mismo como persona, como sujeto que puede ser amado. Mas según se desarrolla la escena, François reta su neurosis de abandono mediante disalienation -desalienación. En Peau noire, Fanon propone la desalienación como un modo de resistencia al discurso de exclusión racial colonial. El filósofo 
explica que "the effective disalienation of the black man entails an immediate recognition of social and economic realities" (FANON, 2008, p.4). Es decir, que la desalienación consiste en reconocer y a la vez rechazar una realidad que enajena subjetividades otras. La desalienación es decir no a un discurso deshumanizante, jerárquico, blanco. François cierra la escena con, "Then it's I who doesn't love you" (FANON, 2018, p.87). Ya que Ginette no logra explicar su amor fuera de la lógica emotiva y relacional establecida por ellos, por el sistema racial/colonial, François elige rechazarla y a la vez rechaza un discurso que niega su humanidad como sujeto negro. Al decirle no a su amada, François deja atrás su neurosis de abandono y articula la desalienación de su persona. En esta corta escena, Fanon logra representar la neurosis de abandono que aflige al sujeto alienado por una sociedad que lo desarticula, a la vez que demuestra cómo la desalienación puede combatir dicha neurosis.

En L'Oeil se noie Fanon también indaga en la noción de sociogenia y en cómo la realidad social difiere para sujetos negros y blancos. Según la noción de sociogenia que propone Fanon, el individuo se forja por medio de procesos biológicos y sociales. Ya que la formación del individuo es en parte el resultado de procesos sociales, Fanon argumenta que la racialización se produce con base en estructuras sociales. Tomando en cuenta la noción de sociogenia que defiende Fanon, sugiero que la realidad negra y la realidad blanca no son iguales. En una sociedad blanca, antinegra, el discurso racial colonial excluye al sujeto negro de la realidad social existente y lo blanco se legitima como moneda de cambio para la articulación de humanidad y agencia. Segun Fanon, "The only means of breaking this vicious circle that throws me back on myself is to restore to the other, through mediation and recognition, his human reality, which is different from natural reality" (FANON, 2008, p.169). Propongo que lo que Fanon identifica como la realidad natural es la realidad racial/colonial del sistema colonial moderno. En esta realidad racial/colonial, el sujeto blanco es humanizado y autorizado, y el negro es violentado y deshumanizado. Al ser alienado por la realidad racial/colonial, el sujeto negro experimenta una realidad distinta a la realidad del blanco. La alienación que producen estas realidades paralelas conlleva al sujeto negro a la búsqueda de reconocimiento, es decir, a la búsqueda de una realidad humanizadora que legitime su existencia. En esta obra de teatro, Fanon juega con esta doble realidad — negra/blanca — a través de las perspectivas que representan los hermanos —François/Lucien. 
Antes de analizar las diferentes realidades descritas en el texto es preciso analizar cómo paso a identificar a François y Lucien como sujetos negro y blanco, respectivamente, ya que como explica Young la obra no identifica a los personajes racialmente. Aunque Fanon elige no nombrar la raza de los personajes, el juego de luces y el simbolismo de los nombres me llevan a asociar a los personajes masculinos a una raza específica. Al situar a los personajes entre luces y sombras, el autor apunta a la raza de los mismos. Las anotaciones indican que Lucien debe ser iluminado por "pewter colour" (FANON, 2018, p.81). Es decir, por una luz plateada. Por su parte, François es iluminado por un "new blotting paper colour" (FANON, 2018, p.81). Young argumenta que, "since new, therefore unstained with dark ink" (YOUNG, 2018, p.33). Aunque las luces mismas no indican la raza de los personajes, propongo que al iluminar a los personajes con estos colores Fanon sugiere que Lucien es blanco y François es negro. El estaño varía en diferentes tonos de grises, al iluminar a Lucien con este color Fanon sugiere que el contacto del gris con la piel blanca del personaje crea un aura plateada, crea luz la cual simboliza lo blanco. En el caso de François, al indicar específicamente que el color que lo ilumina es de papel secante y no simplemente de papel sugiere que François es la marca negra en medio del papel secante que es el escenario. Mediante las luces que iluminan a los personajes, Fanon indirectamente insinúa la identidad racial de los hermanos.

Por otro lado, el autor también expresa la identidad racial de François y Lucien por medio del simbolismo de sus nombres, particularmente en el caso de Lucien. François, proviene del latín y significa hombre libre. A pesar de que hombre libre no hace referencia específica a una raza o color, históricamente, en especial durante la era colonial europea de las Américas, el termino hombre libre se asocia al esclavo negro que ha obtenido su libertad. Aunque quizás en el caso del nombre de François me este extralimitado en buscar simbolismos, el nombre de Lucien claramente sugiere que es un sujeto blanco. Lucien proviene de la palabra latín lux que significa luz. La luz es comúnmente representada por el color blanco, lo cual indica que Lucien puede ser entendido como un sujeto blanco. Al igual que con el juego de luces, el simbolismo de los nombres de los hermanos insinúa la identidad racial de los personajes.

Más allá del significado de los nombres y de una lectura racial de las luces, la relación con la realidad que sostienen los personajes sugiere la posible raza de ambos. El conflicto existencial 
que presenta el texto habla sobre dos posibles relaciones con la realidad: el hedonismo o el absurdisimo. Teniendo en cuenta los demás textos de Fanon, en particular Peau noire, este dilema existencial entre el hedonismo de Lucien y el absurdisimo de François representa las posibles realidades que el colonialismo crea para el sujeto blanco y el sujeto negro, respectivamente. Según la noción de sociogenia que propone Fanon, la sociedad desempeña un papel importante en la formación del individuo. Considero que Lucien y François simbolizan cómo el sistema de violencia y racialización que produce el colonialismo conduce a dos tipos de realidades; una para el colonizador blanco y humanizado, y otra para el colonizado negro ${ }^{4}$ y deshumanizado. Según la narrativa, un extraño episodio con una estrella es lo que desvía el camino de los hermanos. Lucien explica:

And then there was that star that interfered with it all... [...] I had seen a star. / I swear to you that I saw it, Ginette. It was Green with fire. To François I said, look at the star. He stared for a long time at the spot I showed him but the star had disappeared. He then looked at me with wet eyes. / He cried for a long time. He stayed in his room for three days. He has not spoken to me since (FANON, 2018, p.93).

Este episodio con la estrella de fuego no se vuelve a mencionar en la obra, pero según Lucien es este momento que cambia por completo no solo su relación con François sino también la relación de François con la realidad. Teniendo en cuenta la importancia de este episodio en la formación de François, sugiero que la estrella simboliza la sociedad y particularmente el encuentro del personaje con una sociedad colonial y racializada. Aunque ambos hermanos ven la estrella, la realidad social de un mundo antinegro solo François - como sujeto negro - es afectado por ella. La estrella como símbolo de una sociedad racial/colonial muestra las diferentes realidades que la sociogenia construye para el sujeto blanco y el sujeto negro.

La realidad del sujeto blanco en un mundo antinegro puede ser una de hedonismo. Al igual que Lucien, el sujeto blanco dentro de un sistema racial/colonial puede disfrutar de su vida sin mayores preocupaciones sobre la muerte. La visión de Lucien de vivir con un enfoque en el placer y lo estético, representa una existencia que no tiene que tomar en cuenta la violencia del

\footnotetext{
${ }^{4}$ Cabe indicar que, aunque en este texto me enfoco en la dicotomía racial negro/blanco, el sistema racial/colonial también construye jerarquía de humanidades que oprimen a otros sujetos colonizados y racializados, entre ellos indígenas y asiáticos. Partiendo de la noción de "Dark Other" que propone Lewis R. Gordon, el enfoque no sugiere que solo sujetos epidérmicamente negros son alienados por el discurso racial/colonial sino todo sujeto que es identificado como el "Dark Other".
} 
colonialismo o la alienante realidad racial. Como sujeto blanco, Lucien puede enfocarse en los placeres que proporciona el mundo y no en las limitaciones y la violencia que lo construyen. El encuentro con la estrella, con la realidad racial/colonial, no representó ningún trauma para Lucien porque la sociedad con la que se encuentra es una que legitima su humanidad. Lucien dice, "You see, I've thought hard about all that: I think ultimately that $\operatorname{man}^{5}$ will always have to choose between life and death. It said that man is great because he accepts to die. But dying is nothing. The greatness of man lies perhaps in his acceptance of life" (FANON, 2018, p.96). La sociedad antinegra colonial le permite a Lucien, como hombre blanco, elegir cómo vivir y garantiza su protección contra una muerte violenta. Son estos privilegios de blanco lo que autorizan a Lucien a creer que él puede "seize life with both hands, head-on like a gladiator" (FANON, 2018, p.97). Mas en un mundo antinegro, el sujeto negro no es considerado hombre y en esta falta de humanidad su vida o muerte no es una elección personal sino social. Al ser blanco, Lucien puede elegir el hedonismo como filosofía de vida ya que su estatus de humano garantiza la libertad y seguridad que le permite soñar "that I was rich, famous, loved" (FANON, 2018, p.102).

Por su parte, François simboliza al sujeto negro colonizado que está consciente de su racialización y de la realidad en la que el colonialismo lo sitúa, una realidad que lo deshumaniza y lo posiciona en el no-ser. François es un sujeto existencial que ve la vida como algo absurdo. Desde la primera escena, François busca por medio de su relación con Ginette ser reconocido. Al pedirle a Ginette que le explique el por qué lo ama, François - similar al argumento de Fanon en Peau noire - busca ser reconocido como sujeto, como humano. Fanon sugiere que "Now - and this is a form of recognition that Hegel had not envisaged - who but a white woman can do this for me? By loving me she proves that I am worthy of white love. I am loved like a white man" (FANON, 2008, p.45). Al ser amado como un hombre blanco es amado, François es reconocido por Ginette como humano. Fanon afirma, "It is on that other being, on recognition by that other being, that his own human worth and reality depend. It is that other being in whom the meaning of his life is condensed" (FANON, 2008, p.169). Es decir, que es el reconocimiento del otro — de un otro blanco, humano - lo que articula la humanidad del yo. François le pide a Ginette que lo ame, que lo reconozca, pero no como "they call their feelings" (FANON, 2018, p.84). El reconocimiento que busca François sale de las normas establecidas por el discurso racial/colonial. Al contrario del

\footnotetext{
5 Énfasis mío
} 
burgués nacional - como se analiza en Les damné de la terre (1961) — François desea un reconocimiento no europeo, no colonial. El encuentro con la estrella le reveló a François no solo la realidad racial/colonial sino también su posición como sujeto inferior en la jerarquía de humanidades que crea el mundo antinegro. Consciente de que él "would never succeed in gaining recognition" (FANON, 2008, p.43) en un mundo antinegro, François “[...] resolved, [that] since it was impossible for me to get away from an inborn complex, to assert myself as a BLACK MAN. Since the other hesitated to recognize me, there remained only one solution: to make myself known" (FANON, 2008, p.87). En este "make myself known," Francois rechaza el mundo antinegro que lo deshumaniza y busca construir una realidad distinta a la de ellos.

En Peau noire, Fanon argumenta que "The black man possesses two dimensions: one with his fellow Blacks, the other with the White. A black man behaves differently with a white man than he does with another black man" (FANON, 2008, p.1). La constante distinción que François hace entre "they/their" y "I/my", enfatiza que existen dos mundos opuestos: el mundo antinegro de ellos en el cual existen una jerarquía racial y el mundo del yo en el cual la humanidad de todos es respectada, donde la negritud es legitimada (FANON, 2018, p.82-3). Es el encuentro con una sociedad que lo deshumaniza lo que provoca un sentimiento de alienación en François. Como indica la sociogenia, lo social participa en la construcción del sujeto y dentro de una sociedad racial/colonial la construcción del sujeto negro se basa en la negación de su humanidad. Lucien dice que, "François wants to avoid living" (FANON, 2018, p.91). Al contrario de lo que argumenta Lucien, considero que François no está negándose a vivir, sino a vivir bajo normas coloniales que limitan y jerarquizan su humanidad. Este personaje simboliza no la relación disfuncional del sujeto con la realidad sino cómo la realidad colonial conlleva a la alienación del sujeto racializado. Fanon considera que, "to win the certainty of oneself, the incorporation of the concept of recognition is essential" (FANON, 2008, p.169). Ya que en el mundo antinegro el sujeto negro es ininteligible y su reconocimiento imposible, François, junto a Ginette que al regresar con él lo reconoce como humano sin las limitaciones que establece el sistema racial/colonial, se aleja de una realidad deshumanizante, "broke[s) with horror" y camina a los "ABSOLUTE gates/ where life is seized" (FANON, 2018, p.112).

\section{Conclusión}

La labor literaria de Frantz Fanon representa un importante método de estudio para analizar 
las teorías psicológicas, filosóficas y antirracistas que el filósofo desarrolló a lo largo de su vida. En L'Oeil se noie, Fanon indaga en los efectos psicológicos que el colonialismo y la racialización tienen en el sujeto colonial negro. Esta obra de teatro representa la alienación que produce el deshumanizante sistema racial/colonial. Como hemos visto, mediante diálogos, simbolismos y personajes racializados, L'Oeil se noie dialoga con los conceptos que tres años después Fanon elabora en Peau noire, masques blancs. Con este texto, Fanon inicia su búsqueda por entender la realidad colonial y el racismo, y propone un análisis psicológico-social que no tan solo nombra el problema que causa la alienación, sino que también sugiere una solución para la desalienación del “Dark Other" (GORDON, 1999, p.96). En L'Oeil se noie, François dice: "When words scratch each other's eyes out, the only remaining resource is action” (FANON, 2018, p.92). Por su parte, Fanon cierra Peau noire con "My final prayer: O my body, make of me always a man who questions!" (FANON, 2008, p.181). En ambos textos, Fanon propone la acción, el cuestionamiento, como una solución a la alienación. Este ensayo propone una lectura de L'Oeil se noie desde un análisis psicológico de neurosis de abandono y sociogenia y sugiere que esta obra puede ser entendida como un prólogo a las preocupaciones que Fanon analiza en Peau noire, masques blancs y a lo largo de todos sus escritos. En este texto literario - al igual que en sus obras psicológicas, políticas y filosóficas - Fanon explora sus inquietudes ante la realidad de un mundo antinegro y argumenta a favor de la construcción de una realidad dialéctica y humanizada.

\section{Bibliografía}

FRANTZ, Frantz. Black Skin, White Masks. Tran. Charles Lam Markmann. Pluto Press, 2008.

FRANTZ, Fanon. "The Drowning Eye". Alienation and Freedom. Ed. Jean Khalfa y Robert J.C. Young. Tran. Steven Corcoran. Bloomsbury Academic, 2018.

FRANTZ, Fanon. The Wretched of the Earth. Tran. Richard Philcox. Grove Press, 2004.

GORDON, Lewis R. Bad Faith and Antiblack Racism. Humanity Books, 1999.

YOUNG, Robert J.C. "Fanon, revolutionary playwright". Alienation and Freedom. Ed. Jean Khalfa y Robert J.C. Young. Bloomsbury Academic, 2018. 